\title{
Para a crítica da transição nutricional
}

For a critic to the nutritional transition

1 Departamento de Nutrição e Núcleo de Estudos em Saúde Coletiva da Universidade Federal do Rio Grande do Norte. Campus da Saúde. Av. General Cordeiro de Farias s/n, Petrópolis, 59150-000, Natal RN. iliada@uol.com.br

\begin{abstract}
I discuss in this article the characteristics of the nutritional transition. For that, I use the data of nutritional researches of the population, concepts and analytic categories. I make a correlation between these characteristics and the specificities of our development process. Then, I question: 1) The sense of this transition, which comes from a stage initially signed by high prevalence of deficits malnutrition, on gravity forms. 2) If the breakpoint used to reckon the gravity malnutrition occurrences, located under the percentile 3 of the international standard classification - of the National Center of Health Statistics (N CHS) - , is not to choose gravity as hierarchy of the nutritional attention. 3) If this procedure does not mask the real extension of the moderate and light forms of malnutrition deficits. 4) If the selected criterion does not reproduce iniquity in the malnutrition population. 5) If this transition is moving in the direction of improving, optimizing the frame of the Brazilian nutritional situation or making it worst, more complex.
\end{abstract}

Key words Nutritional transition, Gravity, Chronicity, Complexity
Resumo Discuto neste artigo as características da transição nutricional. U tilizo dados dos estudos nutricionais de população, conceitos e categorias analíticas. Correlaciono essas características com as especificidades do nosso processo de desenvolvimento. Questiono o sentido dessa transição que migrou: 1) De um perfil inicialmente marcado pela alta prevalência da má nutrição por déficits, nas formas graves de desnutrição energético- protéica e carências específicas. 2) Se o ponto de corte utilizado para contagem dos desnutridos graves, situados abaixo do percentil três do padrão de classificação do NCHS (N ational Center of $\mathrm{H}$ ealth Statistiscs), ao eleger a gravidade como hierarquização para a atenção nutricional, não mascara a prevalência do déficit de peso e de estatura da população estudada. 3) Conseqüentemente, a real extensão das formas leves, moderadas e a cronicidade da desnutrição. 4) A reprodução da iniqüidade entre os desnutridos. 5) Se essa transição traz em sua trajetória as características de otimização ou de complexificação da situação nutricional da população brasileira.

Palavras-chave Transição Nutricional, Gravidade, Cronicidade, Complexidade 


\section{Introdução}

Este artigo consiste num exercício de reflexão epistemológica na medida que traz à tona pressupostos filosóficos de métodos ou resultados de investigações científicas da atualidade e o entendimento de que, para realizar um trabalho intelectual produtivo, a única maneira possível de fazê-lo, é tentando (Bunge, 1980). Nesta direção, resgato avaliações da situação nutricional de população e comento a evolução dos dados que descrevem a chamada transição nutricional. Teço considerações sobre o sentido e as características ocorridas no processo dessa transição, tendo como fundamentação teórica para a questão alimentar enutricional brasileira a teoria da determinação social da doença. Por esta opção, e movida pelo debate contemporâneo acerca da complexidade, destacados em M inayo (2001) e M orin (1994) entre outros, utilizo conceitos e categorias analíticas básicas, já cristalizadas no referencial teórico que dão suporte aos meus questionamentos e considerações.

Entre esses, o questionamento central que aqui formulo refere-se ao procedimento metodológico atual de avaliação nutricional, quanto à utilização do ponto de corte no percentil 3 do padrão de classificação utilizado, como critério de elegibilidade e contagem dos desnutridos no sistema formal de saúde. Entendo que esse critério resulta no mascaramento das formas intermediárias (leves e moderadas) da desnutrição energético-protéica. Argumento contrário a este seria o de que, "ao incluir-se os situados entre o percentil 3 e 10 resultaria na superestimação do quadro com falsos-positivos", segundo Batista Filho (1994). Considero que o citado procedimento se origina em dilemas e contradições existentes entre a epidemiologia, a clínica e o social, apontados em Almeida (1990). Em si, trazem o peso da cultura hegemônica positivista do empirismo clássico, tais como o risco epidemiológico como critério de elegibilidade na hierarquização das formas de vida e de morbi-mortalidade para priorização da aten ção médica.

A opção de inversão do biológico pelo social concebe que este critério de avaliação reproduz a iniqüidade na ordem sanitária e social ao excluir os excluídos, já biologicamente inferiorizados. Usando a gravidade como limite para a seleção e atenção nutricional, el ege o risco epidemiológico e hierarquiza os famintos, ocultando as formas intermediárias da desnutrição e a cronicidade. Reforça tabus e estigmas, fortemente cristalizados na formação social brasi- leira como a fome endêmica e sua irreversível conseqüência no padrão nutricional da população, o nanismo nutricional, dantes sistematizados em Castro (1983), Chaves (1985) e elliffe (1968).

\section{Eufemismos da fome endêmica}

O conceito de má nutrição aqui adotado segue a trilha da teoria de determinação social da doença, num nível mais geral do processo de determinação social, segundo a qual, biologicamente, a má nutrição é resultante do déficit ou excesso, na pauta dietética, de nutrientes essenciais e de alto valor biológico: as proteínas, hidratos de carbono e gordura. Secundando Costa (1982), Baldijão (1979), Ypiranga (1982), M inayo (1986), Valente (1986) e Escoda/Lima (1989), as formas de má nutrição resultam em função do consumo das forças de trabalho no processo social de produção. Tendo como bases essas referências, a desnutrição energético-protéica, aqui discutida como problema de saúde coletiva para o setor da saúde pública, significa a manifestação biológica da fome endêmica. Esta por seu turno é resultante do processo social de exploração que, nos pontos extremos do consumo, realiza uma pauta dietética deficitária ou excessiva em hidratos de carbono e proteínas de baixo valor biológico e de custo. Seguindo essa lógica, têm diferentes etiologias as formas da má nutrição. Por déficit alimentar, manifesta-se na desnutrição energético-protéica, nas carências vitamínicas e minerais: a hipovitaminose $A$, o bócio endêmico e a anemia ferropriva. Por excesso alimentar, nas dislipdemias, na obesidade e no diabetes II. Socialmente de terminadas, constituem problemas de saúde pública, segundo os critérios do setor, pela sua magnitude descrita nas elevadas taxas de prevalência em que ocorrem, nos danos biológicos e sociais que acarretam, e, sobremaneira, nas possibilidades de reversibilidade disponíveis nos níveis assistenciais, tecnológicos e econômicos.

Dentre as formas de má nutrição por déficit, a desnutrição está epidemiologicamente escalonada em graus de gravidade e cronicidade. A severidade em suas formas clássicas são o marasmo e o kwashiokor. Depende de variáveis como a idade, natureza, duração e intensidade da deficiência dietética. Isto significa dizer que quanto mais precocemente se instalar a fome endêmica, como por exemplo na vida gestacional 
com elevado déficit protéico de origem biológica e calórica, em baixos níveis de adequação às necessidades nutricionais e de longa duração, mais severa será sua manifestação clínica expressa em graus, sinais e sintomas. A desnutrição, também chamada subnutrição, ambas eufemismos da fome endêmica, é clássica tipificação de endemia nos termos definidos em Rouquayrol (1994).

A gravidade e a cronicidade da desnutrição têm como variáveis universais para a sua descrição, o peso e a altura em relação à idade. Esses indicadores são universal mente utilizados por sua razoável fidedignidade, adequação ao setor da saúde pública e sensibilidade. 0 sobrepeso consiste em exceder um padrão baseado na altura e expressa-se no índice de massa corporal. Portanto, a obesidade consiste no aumento ponderal acima do ideal para a altura individual ou coletiva produzindo gordura excessiva, enquanto a altura reflete o crescimento somático como um todo. 0 peso, ao contrário da altura, depende menos de proteínas do que de lipídeos e hidratos de carbono de alto valor biológico. Pode atingir a normal idade relativa à altura sem o rigor da natureza protéico-calórica. A exemplo, em função do excesso de consumo de hidratos que fornecem calorias vazias, ocorre extravasamento do líquido intersticial produzindo edema que resulta num peso de fal sa nutrição. Por esta razão fisiológica é que as categorias analíticas universais diretas do estado nutricional e de desenvolvimento populacionais mais sensíveis são as variáveis: altura $X$ idade, seguida de peso $X$ idade e, por último, peso X altura (Waterlow \& Alleyne, 1974; Waterlow 1976).

Estes autores comprovam a gravidade da desnutrição comparando o déficit de peso de uma criança em relação a uma outra de peso normal, de mesma idade e de classe social superior. Por outro lado, para comprovar a cronicidade da desnutrição esses autores o fazem por meio do déficit estatural de uma criança em relação a uma outra de altura normal, de mesma idade e de classe social superior. Incontestavelmente, uma das formas diretas de se atestar a desnutrição em suas manifestações clínicas éo método antropométrico.

Sobre o conceito e operacionalização de categorias analíticas do estado nutricional da população, Vasconcelos (1993) os sistematiza, didaticamente, nos diversos padrões de classificação existentes. Segundo este autor, a compreensão de estado nutricional deve ser busca- da a partir da identificação e análise de suas de terminações estruturais que correspondem aos processos mais gerais da organização social.

0 padrão de classificação nutricional antropométrico utilizado nos estudos e nos serviços de saúde do NCHS (1977) éo indicado pela O rganização M undial de Saúde (OM S). Esse padrão foi construído com base no status nutricional da população de Boston, cuja renda per capita se situa entre as mais altas do mundo. A renda otimizada é uma variável que possi bilita o desenvolvimento do potencial biológico de crescimento somático da altura em relação à idade (Escoda \& Pinheiro 1997). 0 potencial desta forma de crescimento é o mesmo para todas as raças, dependendo da classe social, determinante da renda. Constitui-se no indicador biológico mais sensível para avaliação de desenvolvimento nutricional de população.

\section{Perfilando a transição}

O Brasil, apesar de ser considerado emergente em função do seu considerável grau de desenvolvimento econômico, científico e tecnológico, situa-se entre os países de maior prevalência em deficiências nutricionais. Em 1988, em carta aberta ao presidente da república assinada por estudiosos e pel as entidades nacionais da área de nutrição, tomando como base os dados do Estudo Nacional de Despesa Familiar (ENDEF, 1974), foi reafirmado que: dois terços das famílias brasileiras consumiam uma dieta de baixa adequação calórica e que esse consumo significava uma situação de fome crônica em vários graus de intensidade e sob diferentes combinações de deficiências nutricionais qualitativas e quantitativas.

0 termo transição pode ser entendido como 0 ato ou efeito de transitar. 0 verbo indica um trajeto, uma trajetória. Passagem de um lugar, de um assunto, de um tom, de um tratamento etc., para outro. Mudança de fase num sistema, fazer caminho, passagem ou percorrer (Ferreira, 1988). Para a epidemiologia, a transição consiste na existência de um primeiro estágio que fora marcado pelas doen ças pestilenciais e pela fome epidêmica; um segundo, pelo declínio das pandemias; e um terceiro, caracterizado pelas doenças degenerativas e produzidas pelo homem (Rouquayrol, 1999). Portanto, a mudança de um padrão epidemiológico para outro.

A transição, aqui entendida como processo, supõea evolução e/ou a distensão - de um regi- 
me de governo - movida por forças sociais no interior de um sistema político. Num sentido mais amplo, a transição social corresponde ao estágio evolutivo das forças produtivas determinando a forma de organização social, de condições de vida e de progresso real. Pressupõe que a natureza dos meios de produção interfere diretamente na forma como os homens produzem e consomem os bens dessa produção. A reprodução social, portanto, consiste na qualidade de vida e não na mera reprodução da existência física dos indivíduos ( $M$ arx \& Engels, 1985).

Nesse sentido, a transição nutricional brasileira em curso reside na mudança do padrão de estado nutricional ocorrido e tem como características a complexificação desse quadro por configurar-se desigual e combinado. Até a década de 1970, relacionando-se a evolução dos ciclos econômicos dos modelos agro-exportador e de desenvolvimento urbano-industrial o mapeamento da fome realizado por Josué de Castro -, o quadro nutricional esteve fortemente marcado por surtos epidêmicos de fome. Sua endemia estava geográfica e socialmente localizada no semi-árido e zona da mata nordestina, e na região amazônica, com elevados índices de prevalência da severidade e da gravidade da desnutrição energético-protéica, além de carências nutricionais específicas do complexo $B$, anemias, bócio e hipovitaminose A. As regiões sul, sudeste e centro-oeste foram consideradas áreas de subnutrição e de carências.

Nos anos 80, a situação nutricional era de carência global de nutrientes e de fome generalizadas, ligadas ao oligopólio industrial e financeiro e, com inversão do perfil populacional rural, marca sua incidência nas classes sociais da periferia dos grandes centros urbanos. Nos anos 90 , seguindo o curso do liberalismo, a situação nutricional tem a considerável redução da prevalência das formas graves da desnutrição e do bócio en dêmico, a manutenção dos níveis anteriores de cronicidade e das carências citadas, agregando a obesidade alimentar, 0 diabetes II e as deslipdemias. Para a leitura dos processos ocorridos na situação nutricional e alteração do padrão que conferem as características da transição, utilizo os dados e análises dos estudos nutricionais de abrangência nacional e regional de maior significância: 1) o EN DEF, 1974; 2) a Pesquisa Nacional de Saúde e Nutrição (PN SN), 1989; 3) a Pesquisa Nacional de Demografia e Saúde (PNDS) com seu desmembramento regional (SE-NE); 4) a Pesquisa de
Padrões deVida (PPV), 1996-1997; série de estudos M S/IBGE, 1970-1999.

Como dados mais rel evantes desses estudos, destaque-se que o ENDEF registrou um consumo inferior a $1.000 \mathrm{~g} /$ dia de al imentos como indicador direto da fome endêmica no Nordeste. Nessa região, a gravidade da desnutrição energético-protéica situou-se na proporção de $79,5 \%$, e nos estados do Sul $23,2 \%$. O peso da desnutrição, no desencadeamento das infecções em $60,9 \%$ e em $67,0 \%$ na mortalidade infantil tendo em Scrimshaw (1970) e Carvalho da Silva (1983) os registros dessa sinergia. Esses dados inscrevem a desnutrição como a causa básica ou associada das infecções e dessa mortalidade, sobretudo em seu efeito sinérgico na imunidade e como a mais contundente das manifestações clínicas, resultante das condições de vida.

Após a divulgação dos dados desse estudo, pesquisadores orgânicos da área da saúde coletiva e da epidemiologia incorporaram a discussão e a denúncia da situação nutricional brasileira, dentro do contexto mais geral das condições de saúde da população, dos sistemas de serviços médicos e da ordem sanitária como um todo. Essa incorporação reforçou o caráter da complexidade do quadro epidemiológico ea assunção do princípio da integralidadena assistência à saúde. Pontuou a necessidade de reorganização dos serviços e de modelo assistencial adequados à realidade epidemiológica, preconizados na reforma sanitária que se processa, jurídica e institucionalmente, no final da década de 1980, a chamada "década perdida", em termos de não-investimentos e atrasos no setor social.

Nessa época, agrava-se a ordem sanitária na medida que o perfil epidemiológico combina as doenças chamadas modernas (acidentes de trabalho e de trânsito, neoplasias, doenças profissionais, mentais, a Aids e distúrbios cardiovasculares) com as denominadas doenças do atraso, por se arrastarem desde o primeiro estágio epidemiológico (desnutrição, Chagas, esquistossomose, malária e o mal de Hansen, que elevara sua prevalência). Estas, somadas às doenças diarréicas ocorridas na infância em decorrência da desnutrição, pelas condições de habitação e saneamento básico, tiveram a incidência estimada em 100.000 casos/ano de doenças evitáveis por imunização, e a não-resolutividade de $80 \%$ dos casos da demanda global dos serviços de saúde. A conjugação de diferentes estágios de morbidades revela a mudança no padrão epidemiológico em extremas desigual dades sociais e regionais expressas nos indicadores de 
saúde. Complexifica a realidade epidemiológica e, desta forma, confere a característica mais visível da chamada transição nutricional que éa complexidade.

O cenário econômico-social agravou-se e, conseqüentemente, as condições de vida e os níveis de saúde de largas parcelas da população. U ma resultante epistemológica dessa nova ordem foi a necessidade de reorganização do sistema e do modelo assistencial em saúde. 0 quadro sanitário descrito guarda relação direta com as especificidades do processo de desenvolvimento econômico brasileiro, desigual e combinado no sentido de conjugar e combinar doenças do desenvolvimento e do atraso em discrepantes níveis regionais e de classe. Eleva os custos da assistência na medida que demanda tecnologia simplificada e de ponta, racionalidade de gestão e adequação de modelo assistencial para uma realidade epidemiológica complexa.

As desigualdades em saúde à época chegaram a até 30 anos de diferença entre a esperança de vida de um nordestino para um sulista de mesma classe social, numa média nacional de 67 anos. Na década de 1960, os relatórios anuais do Unicef e do IBGE registram que a mortalidade infantil no país chegou a 115 para cada mil crianças que nasciam vivas e a taxa de mortalidade para menores de cinco anos era de 160 . Em 1987, essa taxa cai para 64 e 87 respectivamente. Em 1990, a mortalidade infantil teve uma média nacional registrada de 48. Em 2000, a mortalidade infantil no Paraná é de 14 contra 95 em municípios do NE/Sergipe e superior a 100 na periferia de $N$ atal, para uma média regional de 74 enacional de 35 que se situa em 34 , em 2001. Evidencia um forte declínio da média. $N$ as regiões norte e nordeste, o dobro da taxa das regiões sul e sudeste. Nos chamados "bolsões de pobreza", a manutenção dos níveis críticos dessa taxa para as mesmas classes sociais.

A queda da mortalidade infantil aqui observada, não se explica pela melhoria das condições materiais de vida da população como um todo em termos de renda, alimentação e moradia. A este respeito, 0 I ndice de Desenvolvimento $\mathrm{Hu}$ mano (IDH), da Unesco (2001), de limiar 500, aponta o valor 543 para municípios como Acari, no sudeste, e as iniqüidades dos níveis de desenvolvimento apontadas nos extremos desse índice entre os municípios brasileiros, sobremaneira na área do mapa da fome no Nordeste. Esteíndiceé, por excelência, o elenco de indicadores de desenvolvimento atualmente utilizado, que serve para determinação de critérios de prioridade das ações de intervenção das políticas públicas.

O declínio dessa forma de mortalidade reflete uma maior e efetiva cobertura dos serviços de saúde, de suas ações internas e externas ao setor. No desenvolvimento dessas ações, considere-se o inestimável peso de ações - realizadas intra e extra-setor saúde pelas organizações não-governamentais - de maior cobertura vacinal, de água de abastecimento, de esgotamento sanitário, queda da fecundidade, campanhas de reidratação oral, de estimulação ao al eitamento materno, de maior controle das endemias e da el evação dos níveis de escolaridade materna. A mortalidade infantil, portanto, não é mais considerada indicador de pobreza. As peculiaridades das características da construção do projeto de saúde pública nacional demonstram como o saber e a prática médica incorporam tecnologia adequada e conferem resposta efetiva à saúde sem que se altere a estrutura social (Luz, 1982).

Considero importante destacar que, historicamente, a mortalidade infantil nacional encontra-se acima da média observada para alguns países da América Latina. 0 Índice de Desenvolvimento Infantil - IDI - (Unicef, 2001) corrobora esta afirmativa. A descrição da mortalidade expressa em média amalgama a realidade otimizada de saúde de uma minoria, aproximando-a aos níveis de Primeiro M undo, mascarando uma iníqua realidade sanitária de classe e região. A média nacional, mesmo que tenha rebaixado significantemente, ainda é considerada elevada para os padrões internacionais de determinação de níveis críticos, uma taxa acima de 10. Não é de todo desprezível que ainda persiste o estigma do sub-registro para o evento do nascimento e da mortalidade infantil nacionais.

A situação nutricional brasileira requer uma avaliação diagnóstica global, direta, sistematizada. Atualizada, monitorizada, tratada e referenciada por um sistema de vigilância à saúde/ nutricional formal, permanente e de ação. Não apenas o mero registro das ocorrências, sobretudo que sinalize o prognóstico da real situação de saúde e nutrição da demanda hierarquizada, em todas as formas da má nutrição com integralidade e eqüidade à demanda hierarquizada. A esse respeito, em debate realizado pela TV Senado (2001), o representante do Comitê da Cidadania pela fome afirmou que a desnutrição no Brasil é de $21,12 \%$. Contrapondo aos níveis de tolerância da OM S/FAO, que são de $10 \%$, acrescentou sobre a vigilância nutricional que 
"o funcionamento do Sisvan, onde existe, éfrio e burocratizado". No mesmo debate, o representante da FAO disse que a desnutrição compreende 800 milhões de famintos, dos quais 45 milhões estão no Brasil.

Pontuando, os estudos nacionais de avaliação nutricional, embora esporádicos, têm relativa aproximação metodológica. Os que primeiro descrevem a desnutrição como manifestação biológica de determinação social remontam à década de 1930, comprovam a gravidade da situação nutricional das classes trabalhadoras em famílias operárias na cidade do Recife e São Paulo. Castro \& Davis (1932) e Viacava et al. (1983) realizaram uma vasta análise dos estudos realizados até então. Uma das considerações contidas nessa referência é a da discussão do ponto de vista epistemológico das características do padrão epidemiológico, dos procedimentos metodológicos utilizados para contagem e seleção dos desnutridos, sobremaneira, dos estudos ocorridos nas duas décadas anteriores à transição nutricional que se esboça nos anos 90.

Destaco que o ponto de corte no percentil 10 do padrão de classificação nutricional, considerado ideal para surpreender as formas anteriores à gravidade da desnutrição, esteve adotado pelo M inistério da Saúde e utilizado no cartão da criança, até meados da década de 1990. Argumento técnico contrário a esse procedimento aqui comentado alega a probabilidade de ele implicar falsos-positivos. A esse respeito, considero que a pertinência ou não do argumento reside na explicação de Castro Rugani (1999). Esta autora considera que não el eger para seleção e atenção nutricional os levee moderadamente desnutridos explica-se no fato de que são irreversíveis e/ou inócuas, em termos de impacto nutricional, as intervenções das ações internas do setor saúde para com esses desnutridos. Não seriam visíveis em curto prazo as efetividades dessas ações. Na minha compreensão, a explicação consiste no sentido do pragmatismo e da reprodução da iniqüidade subjacente, num sistema de saúde que se autopostula universal e equânime, cujas práticas, mais que um princípio, requerem a integralidade das ações em função da complexidade e combinação do quadro de morbidade que está sinergicamente movido pelas síndromes do excesso de consumo, das pluricarências, das degenerativas e das infecciosas.

Das análises realizadas por M onteiro (1991 e 1999), Batista Filho (1999) e outras abaixo citadas sobre a evolução da situação nutricional brasileira e sua transição, destaco as seguintes conclusões:

- a discrepância dos indicadores de desenvolvimento econômicos e sociais entre os estados do sudeste e nordeste;

- com relação aos indicadores nutricionais dos anos 70-90, a "estabilidade de baixas proporções de crianças menores de cinco anos com sobrepeso e a enorme queda da desnutrição";

- a redução da prevalência nacional do baixo peso nessa faixa etária;

- o importante aumento da obesidade em homens e mulheres da população adulta e sua similar evolução nas regiões sudeste e nordeste;

- segundo a renda, as similitudes de prevalência e tendência da obesidade são iguais, com a observação de tendência de queda na população feminina de alta renda do sudeste, cuja explicação precisaria de melhor conhecimento de outras variáveis de desgaste energético e de maiores aprofundamentos na análise;

- o déficit de crescimento na população infantil é uma questão que permanece como problema de saúde pública, apesar da significante queda de sua prevalência;

- a redução da prevalência de $12 \%$ do baixo peso ao nascer para uma manutenção entre $8 \%$ e $10 \%$ dessa taxa.

A obesidade alimentar é a nova mazela com duas etiologias distintas - considerada problema de saúde coletiva pela elevada prevalência. A mbas são socialmente determinadas. Uma, pelo baixo consumo de proteína de alto valor biológico e pelo alto consumo de hidratos de carbono e gordura, incide nas classes inferiores, em bebês ou adultos. Nas populações estudadas, segundo dados da Sociedade Brasileira de Cardiologia (SBC), tem maior prevalência (de até 30\%) em mulheres adultas e de 10\% em bebês e em crianças de até um ano de idade. Resulta, predominantemente, do depósito acumulado pelos hidratos que fornecem calorias vazias originadas de farináceos, açúcares, infusões, macarrões, pães e outros derivados de cereais, de trigo, milho e arroz. Alimentos de mais baixo custo que compõem a pauta de al imentação, associada ao consumo em menor freqüência de leguminosas, frango e embutidos. A explicação etiológica é a da farinização da dieta com produção de edema e, no caso de bebês, a elevação da precocidade da má nutrição protéico-calórica seja na forma de desnutrição ou obesidade.

O utra etiologia é a da obesi dade alimentar das classes superiores, pelo consumo em excesso de infusões, hidratos, proteínas e gorduras 
de alto valor biológico. Distintas da obesidade mórbida de ordem genética, por disfunções endócrinas metabólicas etc., que possibilitam a formação excessiva de tecido adiposo e maior retenção de líquido.

Na última década ocorreram estudos de avaliação nutricional de abrangência regional significativos. Dentre eles, o que foi realizado no Rio Grande do Norte teve caráter de censo com 40.323 crianças menores de cinco anos da rede de saúde, escolar e de extensão agrícola (Escoda/Lima et al., 1994). Os resultados desse censo comprovaram uma cronicidade de $41 \%$ e uma gravidade de $28 \%$ nas crianças de $0-5$ anos. Se gundo o mesmo padrão de classificação utilizado nos demais estudos, o do $\mathrm{NCHS}$, o ponto de corte utilizado em ambos foi para os situados abaixo do percentil 10.

Outro estudo realizado de significância regional foi com os recrutas de 17-18 anos num total de 750 soldados das forças armadas do exército e das bases regionais $\mathrm{NE}$ aérea e naval, em Natal (Araújo, Escoda/Lima et al., 1988). Utilizou-se o já citado padrão e critério de ponto de corte resultando que, mesmo após um ano de serviço militar, $21 \%$ dos recrutas saíram dos quartéis com déficit de peso e $68 \%$ com dé ficit de até $15 \mathrm{~cm}$ de estatura. Estes dados reafirmam a tese da irreversibilidade da cronicidade no nanismo nutricional e de sua elevada prevalência, apesar de um ano, por suposto, de intensa atividade física e com alimentação balanceada durante o período de serviço militar. Observo que, em informe do Estado Maior das Forças Armadas do mesmo período, consta que se exclui na seleção prévia do alistamento para os quartéis os recrutas considerados "biologicamente inaptos" (Escoda/Lima, 1989).

Outro estudo de avaliação nutricional de caráter explicativo, realizado por Schichieri (1999) na cidade do Rio de Janeiro, em 2.040 domicílios, utilizou a variável de consumo alimentar validada, relacionando a renda com a otimização da adequação nutricional. Esse estudo comprovou que o maior problema de inadequação de consumo de frutas, vegetais e leite encontrase na população de baixa renda, daí uma baixa cobertura na adequação de cálcio, vitaminas C e A. N essa mesma direção, o consumo inadequado de gordura total e colesterol.

Historicamente, os estudos de consumo alimentar no país comprovam a otimização da renda com a adequação desta forma de consumo. Isto desmistifica a suposição de que o povo não se alimenta corretamente por ignorância nutricional e, portanto, não preencheria em sua pauta dietética as necessidades em energia: proteínas, carboidratos e gorduras e demais nutrientes essenciais.

0 peso das doenças nutricionais, de distintas etiologias sociais de déficit e excesso de consumo ou biológicas, acrescido ao das doenças cardiológicas, cerebrais, vasculares, neurológicas, neoplásicas, sexualmente transmissíveis, do stress pelo desemprego e pela violência, além das demais endemias seculares que se cronificam, como dengue, malária, peste, leishmaniose, doença de Chagas e 0 aumento da incidência da tuberculose (Sistema de Informação em Saúde, SIS, 2001), compõem a realidade epidemiológica de forma desigual, combinada. M ais para a sua complexidade do que para a evolução progressista do estágio de morbidades, considerando-se a iníqua conjugação dos problemas endêmicos do primeiro estágio com a elevação de prevalência de males do terceiro.

Das manifestações nutricionais elencadas, a que transita em todas as fases em alta prevalência é a cronicidade da desnutrição através do nanismo e das formas intermediárias do baixo peso não mensuradas. A forma grave, apesar de sua considerável redução, tem o dobro do índice de tolerância. A anemia ferropriva, a mais grave e de mai or prevalência entre todas, seguida da hipovitaminose A, não ocorre necessariamente de forma isolada. 0 bócio endêmico oscila na redução de prevalência pela irregularidade da vigilância e da intervenção sistematizada. A epidemiologia analítica nos remete à compreensão de que este elenco de doenças é mero formalismo didático. Elas não incidem, necessariamente, de forma isolada. Neste sentido, o malnutrido pode, por excesso, realizar as formas nutricionais acima descritas e, por deficiência, a desnutrição energético-protéica, as carências minerais específicas, as hipovitaminoses, e as oligocarências: a denominada síndrome pluricarencial (Batista Filho, 2000). As carências aliadas aos excessos e disfunções nutricionais da modernidade e às complicações cardiovasculares requerem os sentidos e a práxis da integralidade, da eqüidade e da universalidade na assistência médica para a pesada agenda do setor da saúde pública brasileira. 


\section{Referências bibliográficas}

Almeida Filho N \& Rouquayrol M Z 1990. Introdução à epidemiologia moderna. Ed. Apce, Rio de Janeiro, 223pp.

Almeida Filho N \& Rouquayrol M Z 1999. Epidemiologia \& Saúde. Ed. M edsi, Rio de Janeiro, 570pp.

Araújo, Escoda/Lima et al. 1988. Avaliação nutricional de ingressos nas forças armadas em $\mathrm{N}$ atal. V Congresso Brasileiro de Nutrição. ASBRAN, Santa Catarina.

Baldijão CEM 1979. A desnutrição e o processo de acumulação do capital. Cadernos de Pesquisa, n. 29. Ed. FCG, São Paulo.

Batista Filho M 1994. Saúde e Nutrição, pp. 365-382. In N Almeida Filho \& M Z Rouquayrol. Epidemiologia \& saúde. 4ạ ed. Ed. M edsi, Rio de Janeiro.

Batista Filho M 2000. Entrevista. In FA Vasconcelos. A epidemiologia das deficiências nutricionais no nordeste. Cadernos de Saúde Pública 16(2). Fiocruz, Rio de Janeiro.

Bunge M 1980. Epistemologia. Ed. USP, São Paulo, 246pp.

Carvalho da Silva A 1983. Desnutrição: nordeste, desespero ou esperança. Ciência Hoje, n. 5. Ed. FAPESP, São Paulo.

Castro \& Davis 1932. Situação nutricional de operários da cidade do Recife e São Paulo. In Escoda/Lima MSQ 1989. A determinação social da fome/desnutrição e a intervenção do Estado. Dissertação de mestrado. Pós-graduação em Ciências Sociais/Sociologia/ Desenvolvimento, UFRN, Natal, 116pp.

Castro IR 1999. Avaliação da operacionalização e impacto do programa de recuperação institucional. Saúde em Foco, n. 18. Ed. SM S, Rio de Janeiro.

Castro J 1983. Geografia da fome. Ed. Antares, Rio de Janeiro, 361pp.

Castro J 1983. Fome, um tema proibido. Ed.Vozes, Petrópolis, 154pp.

Chaves N 1985. Nutrição básica e aplicada, pp. 178-186. Ed. Guanabara, Rio de Janeiro.

Costa OV 1982. A nutrição - má nutrição na reprodução social das classes trabalhadoras: uma hipótese de trabalho, pp. 21. Faculdade de M edicina da USP, Ribeirão Preto (mimeo).

ENDEF 1974. Estudo Nacional de Despesa Familiar, Brasil. Série de Estudos do IBGE.

Escoda/Lima M SQ et al. 1994. Censo nutricional-RN . IV Congresso Brasileiro de Saúde Coletiva, Resumos. Abrasco, Recife.

Escoda/Lima M SQ 2000. Transição nutricional: gravidade e cronicidade. $V$ Congreso Latinoamericano de N utrición, SLAN, Workshop, Buenos Aires.

Escoda/Lima M SQ \& Pinheiro M D 1997. Crescimento e de senvolvimento. Cap. http://www.ufrnet.br/ scorpius. In M anual de Pediatria. Ed. Sociedade Brasileira de Pediatria, Rio de Janeiro.

Ferreira ABH 1988. Dicionário Básico da Língua Portuguesa. Ed. Nova Fronteira, Rio de Janeiro.

Jelliffe DB 1968. Evaluación del estado de nutrición de la comunidad. OM S, Genebra, 291pp.
Luz M T 1982. M edicina e ordem política brasileira. Ed. Graal, Rio de Janeiro, 218pp.

M arx K \& Engels 1985. Da ideologia alemã. In Formações econômicas pré capitalistas. Ed. Paz e Terra, Rio de Janeiro, 138pp.

M inayo M CS (org.) 1986. Raízes da fome. Ed. Fase-Vozes, Petrópolis, 185pp.

M inayo M CS 2001. Estrutura e sujeito, determinismo e protagonismo histórico: uma reflexão sobre a práxis da saúde coletiva. Ciência \& Saúde 6(1), pp. 7-19.

M inistério da Saúde/IBGE 1990 - 98. Série. Pesquisas nacionais sobre saúde e nutrição. Brasília.

M inistério da Saúde/SIS. Evolução de prevalência de morbidades. Série 1980-2001. <http://www.saude.org.br>

M onteiro CA et al. 1991. Saúde e nutrição das crianças brasileiras no final da década de 80. In UNICEF/ INAN/IBGE. Aspectos de saúde e nutrição no Brasil, Rio de janeiro.

M onteiro CA 1999. Evolução do perfil nutricional da população brasileira. Saúde em Foco, n. 18. Ed. SM S, Rio de Janeiro.

Morin E 1994. Ciência com consciência. Ed. Publicações Europa-América, Lisboa.

National Center of Health Statistics 1977. Growth curves for children. Birth-18 years. Vital Health Statistics, Boston.

O Estado de São Paulo 1996. M ortalidade infantil. Jornal, c. A, 14 pp. 15 dez, São Paulo.

Rouquayrol M Z 1994. Epidemiologia \& Saúde. Ed. M edsi, Rio de Janeiro, 527pp.

Schichieri R 1999. Consumo alimentar no município do Rio de Janeiro. Saúde em Foco, n. 18. Ed. SM S, Rio de Janeiro.

Scrimshaw NS et al. 1970. Nutrición e infecciones: su acción recíproca. OMS, Genebra.

Sociedade Brasileira de Cardiologia 2001. O besidade. $<$ http://www.cardiologia.org.br>

Unesco 2001. Índice de desenvolvimento humano. <http:// www.unesco.org.br>

Unicef 2001. Índice de desenvolvimento infantil. <http:// www.unicef.org.br>

Valente FLS (org.) 1986. Fome e desnutrição determinantes sociais. Ed. Cortez, São Paulo, 107pp.

Vasconcelos FAG 1993. Avaliação nutricional de coletividades. Ed. UFSC, Santa Catarina, 146pp.

Viacava F \& Figueiredo CM \& Oliveira WA 1983. A desnutrição no Brasil. Ed. Vozes-Finep, Petrópolis, 199pp.

Waterlow JS \& Alleyne GAO 1974. M á nutrição protéica em crianças. Ed. LPM , São Paulo, pp. 3-42

Waterlow JS 1976. Classification and definition of proteincalorie malnutrition. WHO, Genebra, pp. 530-555.

Willet W \& Buzzard MI 1992. Introdução. Nutritional epidemiology. Oxford University Press, Nova York.

Ypiranga L 1982. Desnutrição: contribuição para a análise de sua determinação. Dissertação de mestrado. Fiocruz, Rio de Janeiro, 147pp. 